\title{
ELECTROPHYSIOLOGICAL ASSESSMENT OF VISUAL PATHWAY FUNCTION IN INFANTS
}

\author{
A. KRISS and I. RUSSELL-EGGITT \\ London
}

\begin{abstract}
SUMMARY
The flash ERG and VEP have conspicuous immature features during the first 4 months following birth. The most marked maturational changes occur in ERG amplitude and VEP latency. Concurrent recording of the skin ERG and VEP provides information which is very useful in helping to arrive at a diagnosis in the young infant with nystagmus who appears to be blind and has a fundus of normal appearance. ERG and VEP features associated with Leber's Amaurosis, congenital cone dysfunction, albinism, optic nerve hypoplasia and unilateral hemisphere dysfunction are described.
\end{abstract}

\section{INTRODUCTION}

Electrophysiological recordings from human neonates clearly show that the retina and neural visual pathway are functional at the time of birth. Both retinal and occipital responses change rapidly in the neonatal period, demonstrating that a considerable degree of maturation is occurring, particularly during the first four months following birth. Histological studies in neonates indicates that the maturational process in the visual pathway involves elongation of rod and cone cell outer segment, ${ }^{1,2}$ foveolar differentiation, ${ }^{3,4}$ thickening of the myelin coating at optic nerve and cortical levels, ${ }^{5,6}$ a decrease in the number of dendritic spines, ${ }^{7}$ proliferation of synaptic connections at the lateral geniculate and visual cortex,${ }^{8,9}$ and increase in cortical volume of primary and secondary visual areas. ${ }^{7,10}$ This article briefly describes normal maturational changes of the neonatal ERG and VEP. It then outlines our technique for concurrent skin ERG/VEP recording and, lastly, dwells on selected clinical conditions to illustrate the usefulness of electrophysiological recordings in helping to identify the condition and the retino-cortical pathway level at which dysfunction may be occurring. Visual electrophysiology can be a great asset to the paediatric clin-

From: Eye Department, Hospitals for Sick Children, Great Ormond Street, London WC1N 3JH.

Correspondence to: Dr A. Kriss, Eye Department, Hospitals for Sick Children, Great Ormond Street, London WC1N 3JH. ician by helping to establish a diagnosis at an early stage. A firm diagnosis is essential when giving an assessment of the genetic risks of recurrence, and gauging visual outcome with its associated implications for organising educational help for the patient and support for parents.

\section{NORMAL ERG CHANGES DURING THE NEONATAL PERIOD}

\section{i. Flash ERG}

Compared with the adult ERG, that from the neonate is markedly attenuated and therefore not as easily discerned. Indeed, the earliest reports claimed that an ERG was not recordable from many healthy newborns. ${ }^{11,12}$ However, later studies using improved amplifier technology, computer averaging and brighter stimuli have consistently described small rod and cone mediated retinal responses at term, and even in preterm infants from about 34 weeks gestational age. ${ }^{2,14,15}$ Horsten and Winkelman ${ }^{2}$ used a Henkes contact lens electrode and found that the scotopic b wave amplitude after 15 minutes dark adaptation measured between 40 and $140 \mathrm{uV}$ in infants recorded on the day of birth or very soon after (under these conditions, adult values are around $300 \mathrm{uV}$ ).

Rod mediated responses mature relatively rapidly over the first three months following birth, and more slowly thereafter, so that by 12 months of age the amplitude of the infant ERG is approximately within $10 \%$ of that of the adult. Fulton and Hansen ${ }^{15}$ recorded corneal ERGs using the DTL conductive thread electrode* and monitored scotopic intensity/response (Naka-Rushton) functions for the 'a-b wave' amplitude in 16 two-month-old infants and nine older infants. They found that neonates had smaller maximal amplitudes ( $\mathrm{V}$ max) and that the relative intensity of light required to give the half maximal amplitude (a measure of retinal sensitivity) was 1-2 log units less than in the adult. However, the rate at which 'b wave' amplitude altered with change in light intensity was similar in infants and adults. Sensitivity reached adult values by about six months and maximal amplitudes were similar to the adult around 12 months.

Grose et al. ${ }^{16}$ studied 30 pre-tem infants in a baby care 
unit. They also used DTL electrodes but recorded under photopic conditions (i.e. mainly cone mediated activity was elicited). The stimulation rate was just under $2 / \mathrm{sec}$ and 30 sweeps were averaged for each trial. In pre-term infants of less than 35 weeks, the 'b wave' tended to be broad and the 'a wave' relatively attenuated. Between 30 and 50 weeks (post-conception age) maturational changes in the ERG were virtually linear. For both ' $b$ wave' amplitude and latency, there was a linear correlation with age (amplitude appeared to increase at the rate of about $3 \mathrm{uV} /$ week and latency decreased by approximately $0.8 \mathrm{~ms} /$ week).

Our department is primarily concerned with the ophthalmological appraisal of infants and young children. Corneal contact electrodes indisputably give the largest ERGs which, compared with other electrodes, are least likely to be affected by physiologic or extrinsic interference potentials. Contact lens electrodes have been recommended as the standard since they provide the most accurate electroretinographic assessment of disease processes involving rods and cones. ${ }^{17}$ However, although the majority of adults will tolerate contact lens electrodes, most young children will not (particularly those between three months and five years or so). Children commonly become tearful and restless when corneal electrodes are inserted and it is often difficult to obtain reliable corneal ERG recordings without sedation or anaesthesia.

We reserve use of examination under anaesthesia with corneal electrodes for exceptional circumstances where a child is too active or behaviourally disturbed to permit placement of electrodes of any type. For routine, screening, assessments, we have opted for recording averaged ERGs using skin electrodes sited centrally under each eye, within $1 \mathrm{~cm}$ of the rim of the lower eyelid. The majority of young children will accept lower lid skin electrodes for the duration of the recording session. $\dagger$ We do not favour routine use of sedation or anaesthesia as these procedures carry risks, and can alter the VEP (the latter can affect the ERG also), thus complicating clinical interpretation of any detected response changes. In our experience, it is diagnostically more advantageous (and cost effective) to average skin ERGs and VEPs together in order to get an estimate of both gross retinal function (from the ERG) and macular pathway function (from the VEP) in a single recording session lasting 30-40 minutes. Flash stimulation is performed while the eyes are open and the child is in a wakened state. In very young infants, the eyelids are held open. A stimulation rate of $3 / \mathrm{sec}$ is used for both flash and pattern stimulation, and each averaging run lasts approximately 40 seconds. By altering the stimulus (flash colour and checksize), we find clinically reliable estimates of retinal cone and rod function, and post-retinal visual pathway function can be obtained (vide infra). In our routine protocol, pattern reversal stimulation under darkened

* The type of ERG electrode used has an important bearing on the size of ERG recorded. Fulton and Hansen ${ }^{15}$ state that the DTL ERG is approximately $60 \%$ the size of that recorded from the Burian-Allen contact lens electrode. We have compared Burian-Allen ERGs with those recorded from DTL, and other type of electrodes, and agree with this relative estimate (Esakowitz, Kriss and Shawkat, in preparation). conditions is tested first; flash stimulation is then performed, first under darkened conditions then photopically. A recording amplifier bandpass of between three and $125 \mathrm{~Hz}$ is normally selected (the low frequency setting is deliberately set somewhat high in order to minimise distortion due to movement potentials).

A bright white flash (Grass PS22, intensity 4, 3.7 $\times 10^{5}$ candlepower) delivered at $3 / \mathrm{sec}$ under fully darkened laboratory conditions elicits a mixed cone/rod ERG. In the young adult, the mixed ERG recorded from skin electrodes has an 'a wave' with a mean latency of $15.6 \mathrm{~ms}$ and mean baseline-peak amplitude of $11 \mathrm{uV}$. The ' $b$ wave' mean latency is $39.3 \mathrm{~ms}$ and the mean ' $a-b$ ' amplitude is $27 \mathrm{uV}$. The skin ERG waveform is broader and smaller in the first few post-natal months. Amplitude and latency changes are rapid during the first four months and slower thereafter until about 12 months. By six months of age ERG component latency and amplitude are approximately within $10 \%$ of that found in the adult. Table I summarises $a-b$ amplitude changes over the first six months for control infants recorded in our laboratory.

Figure 1 shows the averaged flash ERGs, and VEPs, to a bright white flash recorded from the same infant between the ages of 2 weeks and 3.5 years. Note the attenuated, broad ERG in the first 3 months. In all the figures presented both ERG and VEP waveforms are displayed using the clinical neurophysiology convention of 'negativity upwards'.

\section{ii. Pattern ERG}

Fiorentini et al.$^{18}$ monitored skin ERGs to pattern reversal stimulation (P. ERGs) in infants aged between two and six months. A sine wave grating reversing at the rate of 12 reversals/second was used. The ERG and VEP were simultaneously recorded and response amplitude was measured for a range of grating sizes (spatial frequencies). They found that pattern ERGs and VEPs gave similar estimates of acuity and thus concluded that VEP acuity is primarily determined at the retinal level. It has to be said, however, that the spatial tuning of the P. ERG and P. VEP in the adult are not very closely associated. Another point relevant to signal detection and the estimation of acuity electrophysiologically is that when a pattern is progressively defocused, at the point that the pattern ERG becomes non-detectable, the P. VEP is still clearly present and only shows minor change in amplitude and latency. ${ }^{19}$

The small P. ERG recorded from skin electrodes (usually around $2-4 \mathrm{uV}$ ) is susceptible to interference by eye movement potentials and periorbital muscle potentials. We find that in young children, the P. ERG is not as reliable as the flash ERG or pattern VEP in giving clinically useful information.

$\dagger$ Some clinical neurophysiology laboratories record a skin ERG from the bridge of the nose. Lower lid skin ERGs are over twice the size of those recorded from the bridge of the nose and give more accurate monocular information.

$\ddagger$ The averaged skin ERG is about $30 \%$ larger than the monocular pattern VEP, and hence easier to detect. Skin ERGs are approximately $30 \%$ the size of those recorded from a DTL electrode (Esakowitz, Kriss and Shawkat, in preparation) 
Table I. Skin ERG ' $a-b$ ' mean amplitudes ( $\mathrm{uV}$ ) for first 6 months

\begin{tabular}{llccccc}
\hline Age (months) & 1 & 2 & 3 & 4 & 5 & 6 \\
\hline Mean & 9.6 & 13.3 & 18.0 & 29.7 & 24.6 & 27.8 \\
S.D. & 1.8 & 4.6 & 2.0 & 7.2 & 7.1 & 5.5 \\
Number & 9 & 6 & 5 & 3 & 3 & 5 \\
\hline
\end{tabular}

\section{VEP CHANGES DURING THE NEONATAL PERIOD}

\section{i. Flash VEP}

The adult flash VEP has a complex mutlipeak waveform with a characteristic positivity around $90-110 \mathrm{~ms} .^{20.21}$ In contrast, the flash VEP of pre-term infants between 24-27 weeks gestational age has a simplified waveform dominated by negative going activity at about $250-300 \mathrm{~ms}$. Around 32-35 weeks, the positivity characteristic of the adult VEP emerges as a component preceding the dominant negativity and has a latency of $190-230 \mathrm{~ms}^{22},{ }^{23,24,25}$ This main positivity progressively decreases in latency. Grose et al. ${ }^{16}$ report the rate of change was correlated with age (this appeared to be approximately $10 \mathrm{~ms} /$ week between 30 and 50 weeks gestational age). By about four months after a full term birth, latency is within $5 \mathrm{~ms}$ of adult values. Table II summarises maturational latency changes in the main positivity of the flash VEP for control infants recorded in our laboratory. It has been reported that the VEP matures at a faster rate in preterm infants compared with full terms. ${ }^{25}$

\section{ii. Pattern VEP}

Pattern VEPs have been elicited in neonates using miniature TV monitors, standard TV monitors and arrays of light emitting diodes. Harding et $a l^{26}$ and Grose et al. ${ }^{16}$

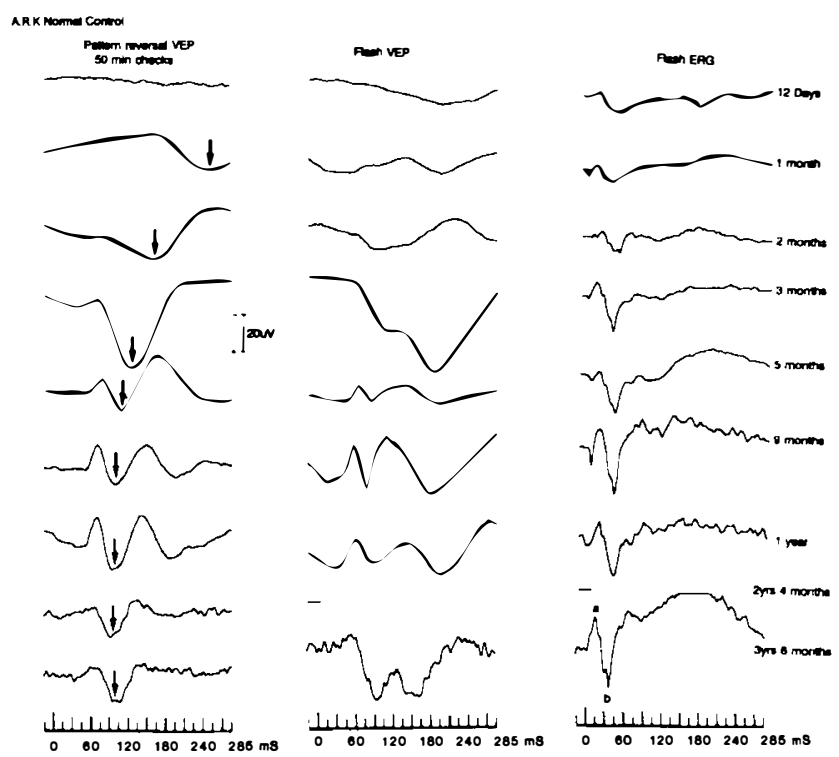

Fig. 1. Pattern VEPs to 50' checks (left column), flash VEPs (middle column) and averaged flash ERGs (right column) from a healthy infant recorded at intervals between 12 days and 3 years 6 months following a normal gestation birth. In particular, note the increase in ERG amplitude and decrease in VEP latency associated with maturation. For this and all subsequent Figures, positivity is downwards. recorded pattern VEPs using a miniature hand held TV. They found in preterms two degree checks gave clearly discernible responses. VEPs matured at an almost linear rate with responses to larger checksizes maturing at a faster rate compared with those to small checks. Moskowitz and $\mathrm{Sokol}^{27}$ have similarly reported that pattern reversal VEPs to 48' and 60' checks are of shorter latency compared with those to $14^{\prime}$ and $12^{\prime}$ ' checks. Table III summarises maturational changes in latency for control infants recorded in our laboratory. The mean data is for the P100 of the pattern reversal response to stimulation with 100 ' checks.

\section{ERG AND VEP RECORDING AS AN AID TO DIAGNOSIS IN THE YOUNG INFANT}

So far, we have carried out routine recordings on approximately 3,000 young children. Our experience covers a wide range of ophthalmological and neuro-ophthalmological conditions. When deciding whether recorded visual responses are abnormal or not, it is vital to take account of the maturational stage of the patient. This important aspect has not always been considered, particularly in studies of Delayed Visual Maturation (see reference 28 for further details).

We nearly always attempt to record VEPs to pattern reversal stimulation in young infants as it gives a more reliable estimate of visual pathway function and of the quality of vision as compared with flash VEPs. The stimulus pattern is presented on a large $26^{\prime \prime} \mathrm{TV}$ display. The infant's attention is directed towards the stimulus pattern by alternating the checkerboard with cartoons or playing nursery rhymes. Fixation is monitored by closed circuit television.

It is important to use checksizes which will elicit conspicuous VEPs, particularly when dealing with young infants. Thus neonates less than three months of age will produce relatively well-preserved, though somewhat late, VEPs to checksizes above 50', but those to checksizes below $25^{\prime}$ are very late, degraded, or simply not detectable $^{27}$ (see Fig. 2).

We have opted to use pattern reversal stimulation for routine clinical testing for several reasons: First, the VEP waveform or component structure does not change with maturation (as compared with pattern onset VEP. ${ }^{29}$ ) Secondly, components associated with stimulation of macular and paramacular parts of the visual field can be identified. ${ }^{30}$ Thirdly, the VEP shows good occipital lateralisation in association with lesions causing hemianopic field defects. ${ }^{31.32}$

What follows is a selective description of ERG and VEP changes in a variety of clinical conditions affecting retinal and post-retinal visual pathway function. The responses presented are all from young infants.

Table II. Flash VEP main positivity. Latency (ms) for first 6 months

\begin{tabular}{lcccccc}
\hline Age (months) & 1 & 2 & 3 & 4 & 5 & 6 \\
\hline Mean & 194.8 & 171.3 & 129.6 & 103.7 & 93.2 & 90.4 \\
S.D. & 11.3 & 29.3 & 35.8 & 12.0 & 4.1 & 8.3 \\
Number & 7 & 6 & 5 & 3 & 5 & 5 \\
\hline
\end{tabular}


Table III. Pattern reversal P100) to 100' checks. Latency (ms) for first six months

\begin{tabular}{lcccccc}
\hline Age (months) & 1 & 2 & 3 & 4 & 5 & 6 \\
\hline Mean & 229.1 & 203.8 & 145.2 & 138.7 & 109.4 & 105.7 \\
S.D. & 22.6 & 44.7 & 31.8 & 21.4 & 5.8 & 4.4 \\
Number & 7 & 6 & 5 & 3 & 3 & 5 \\
\hline
\end{tabular}

\section{i. Leber's Amaurosis}

Figure 3 shows the recordings obtained from a onemonth-old infant with Leber's Amaurosis. The parents were concerned that the infant was not fixing or reacting to bright light. At first presentation to hospital, fundal examination was noted to be normal, pupillary reaction was somewhat sluggish, though not definitely abnormal. The diagnosis was left open for the time being and the patient was referred to our hospital for further assessment and electrophysiological testing (in the referring letter the ophthalmologist expressed the view that the most likely diagnosis was either Leber's Amaurosis or Delayed Visual Maturation). Figure 3 shows that the traces from this patient compared with those of a healthy control at the same age. Note that at this age the ERG and VEP are clearly detectable but attenuated, and that the VEP is markedly increased in latency. The recordings for this patient did not reveal any clinical or biochemical indications to suggest a peroxisomal disorder or a neuroretinal degenerative condition, which can also be associated with poor ERG and VEP activity. In our experience, most Leber's Amaurosis patients $(56 \%)$ have this electrical pattern of no ERG or VEP activity. In $44 \%$ of patients no ERG activity is detectable but a small degraded flash VEP can be recorded (but no pattern VEPs). ${ }^{33}$ This group of patients have some rudimentary degree of vision, at the hand movements or perception of light levels.

\section{ii. Congenital cone dysfunction}

Diagnosis of cone dysfunction in preverbal children predominantly relies on electrodiagnosis. In particular, the demonstration of very poor or absent cone mediated retinal responses tested by using red flash, or photopic testing with a bright white light flashing singly or flickering at rates above 20/s (most commonly tested at 30/s). Patients produce attenuated VEPs to flash stimulation and pattern
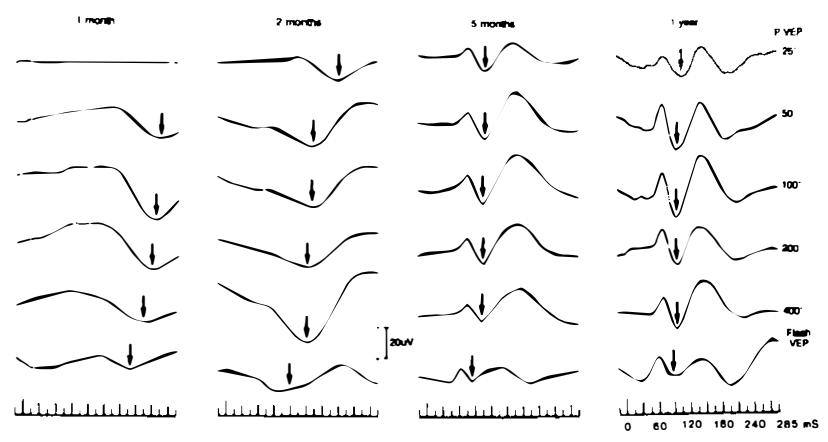

Fig. 2. Pattern VEPs to a range of checksizes in the same infant recorded at $1,2,5$ and 12 months of age. PIO) (arrow'ed) changes in latency with maturation, and the response to the smallest checksize $\left(25^{\circ}\right)$ show's the greatest degree of change. reversal VEPs are commonly not detectable. However, patients who turn out to have partial cone dysfunction (with some rudimentary ability to distinguish between some primary colours) will often produce attenuated, delayed, pattern VEPs.

Figure 4 shows ERG and VEP traces from a 10-monthold male infant who had horizonal nystagmus and photophobia at the time of the recording. He presented to hospital at five months of age and was noted to have unsteady fixation but normal fundal examination and pupillary reaction; photophobia was not apparent at this stage. Electrophysiological recordings showed abnormal cone mediated activity, as reflected in retinal responses to red flash and white flash presented under photopic conditions, and poor pattern VEPs. However, rod mediated activity was well preserved, as demonstrated by responses to dim blue flash and white flash, delivered under scotopic laboratory lighting conditions.

\section{iv. Albinism}

The ERG/VEP picture associated with albinism is very distinct. The albino eye is hypopigmented which leads to ERG differences compared with normally pigmented controls. Anatomical studies of the visual pathway of albino mammals show that nerve fibres from the nasal retina (subserving the temporal visual field) cross at the chiasm in a normal fashion. However, most of those from the temporal retina (subserving the central part of the nasal field) anomalously accompany temporal retinal fibres and cross at the chiasm. Thus, in the albino the majority of fibres from one eye cross at the chiasm and project to the contralateral hemisphere. ${ }^{34}$

These eye and visual pathway anomalies produce ERGs and VEPs with characteristic features. In our experience, using the Grass stimulator at intensity 4 setting, the mixed $\mathrm{rod} / \mathrm{cone}$ ERG of albinos tends to have an accentuated a wave, and $a$ and $b$ waves latencies which are shorter than those of age matched normally pigmented controls. On average, the $\mathrm{a}$ to $\mathrm{b}$ wave amplitude ratio of albinos is 1.3 , as compared with 2.5 in normally pigmented controls. ${ }^{35}$

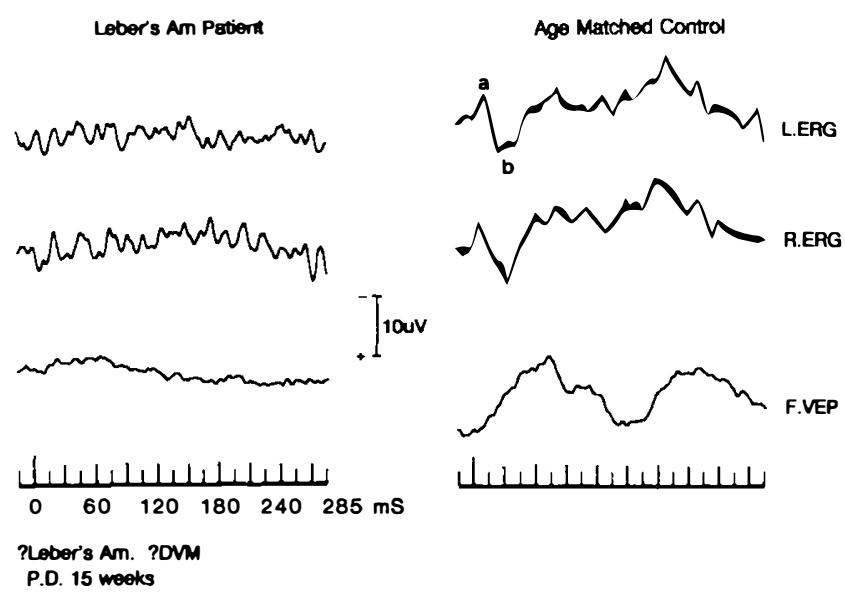

Fig. 3. Comparison of $E R G$ and $V E P$ recordings from a healthy control (on right) and a 15-week-old infant with Leber's Amaurosis. No flash ERG or VEP activity is detectable from the Leber's Amaurosis potient. 


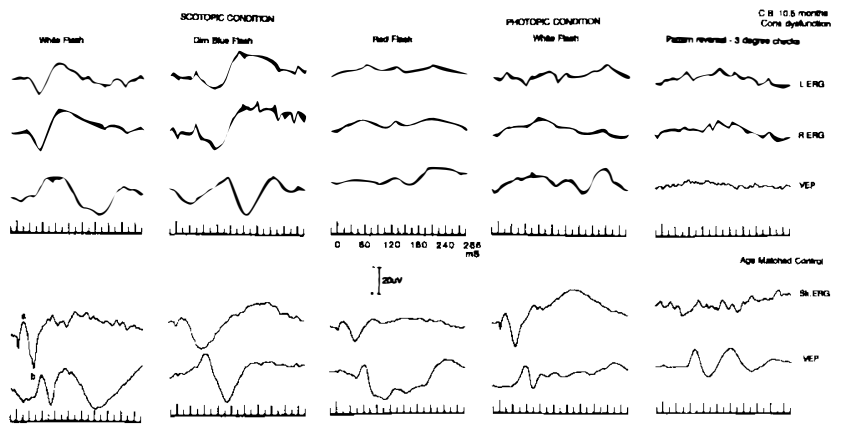

Fig. 4. ERGs and VEPs from a 10.5 month old patient with congenital cone dysfunction (upper traces) and an age matched control (lower traces). Left and right eye ERG and binocular $V E P s$ to white, dim blue and red flash delivered under fully darkened laboratory conditions (the leftmost traces). Also presented are responses to white flash presented under photopic conditions, and response to pattern reversal stimulation. Cone dysfunction patient has well preserved rod mediated ERGs to white and blue flash elicited in the dark, however, those to red flash and white flash delivered under photopic conditions, and pattern reversal VEPs, are all markedly abnormal.

In infants, the flash VEP shows a distinct 'albino' crossed asymmetry on monocular stimulation which is detected at lateral occipital electrode placements.* We find this asymmetry is best seen at a latency of around $80 \mathrm{~ms}$ following the flash. A negativity on the side of scalp contralateral to the stimulated eye and a positivity at about the same latency on the ipsilateral side are usually evident. Young children show the crossed asymmetry of albinism more reliably following flash stimulation than with either pattern onset or pattern reversal stimulation. ${ }^{35,36,37}$ In the adult, however, pattern onset is reported to be the better stimulation mode. ${ }^{37}$

Examination of the mother's fundus can provide useful clinical clues as carriers of the $\mathrm{X}$ linked ocular albinism gene may show mosaic fundal pigmentation, presumably because of the Lyonisation effect.

Figure 5 shows left and right eye skin ERGs and VEPs to flash stimulation (upper pair of traces), and VEPs to pattern reversal stimulation (middle pair of traces) and pattern onset stimulation (lower pair of traces). The mid-occipital electrode (M.Occ) is sited at $\mathrm{Oz}$ (10-20 system), approximately $3.5 \mathrm{~cm}$ above the Inion, and the left (L.Occ) and right (R.Occ) occipital electrodes are laterally placed, half way between the mid-occipital to mastoid distance (about $4 \mathrm{~cm}$ ). All active electrodes are referred to a common mid-frontal electrode at $\mathrm{Fz}$ (10-20 System). The visual responses presented are from a blond three-month-old infant with ocular albinism. At the time of the recording, he had poor fixation and large amplitude pendular nystagmus. Transluscent blue irides and macular hypoplasia were noted on examination. Both his parents had fair hair, and his mother was noted to have mosaic fundus pigmentation typical of carriers of the $\mathrm{X}$-linked ocular albinism gene. The VEP findings are typical of what is seen in ocular or oculocutaneous albino children of this young age. Flash VEPs show the clear crossed asym-

* A VEP crossed asymmetry is also observed in association with chiasmal compression. In this situation, however, the asymmetry is reversed as there is loss of fibres crossing at the chiasm. metry-the occipital distribution is opposite when comparing responses from each eye. Neither pattern reversal nor pattern onset stimulation show similar significant VEP changes.

\section{v. Optic Nerve Hypoplasia}

In severe optic nerve hypoplasia the flash ERG is conspicuous and no VEP activity is likely to be recorded to either flash or pattern stimulation. Interestingly, there is a tendency for the flash ERG to be larger than average. Infants Abinism
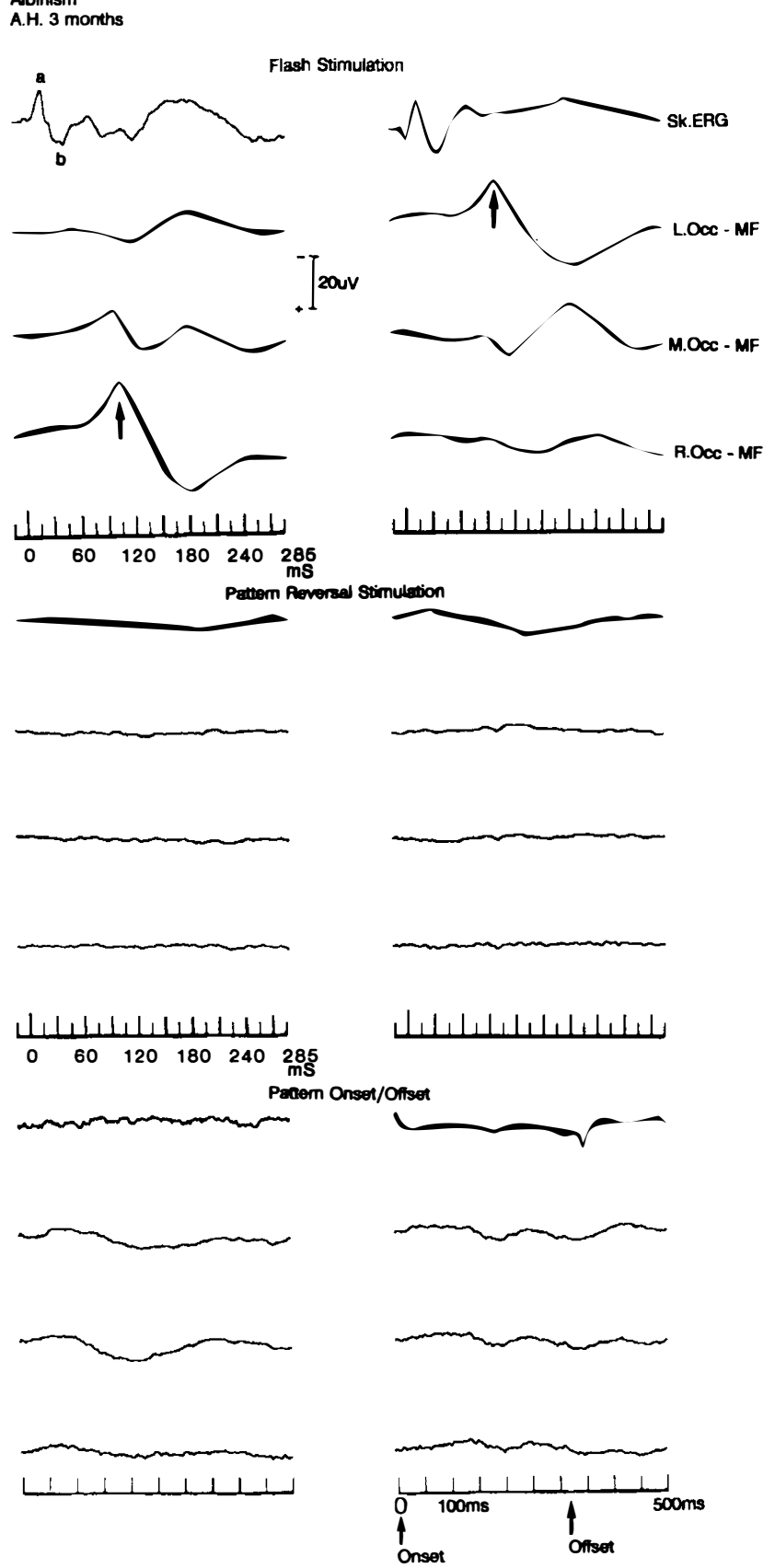

Fig. 5. Flash, pattern reversal and pattern onset response from a 3-month-old infant with X-linked ocular albinism. Traces presented for each stimulation mode are from electrodes under the eye (Sk.ERG), and at left occipital (L.Occ), Mid-Occipital (M.Occ) and right occipital (R.Occ) locations. Note that flash $V E P s$ have a crossed asymmetry when comparing responses from each eye. 


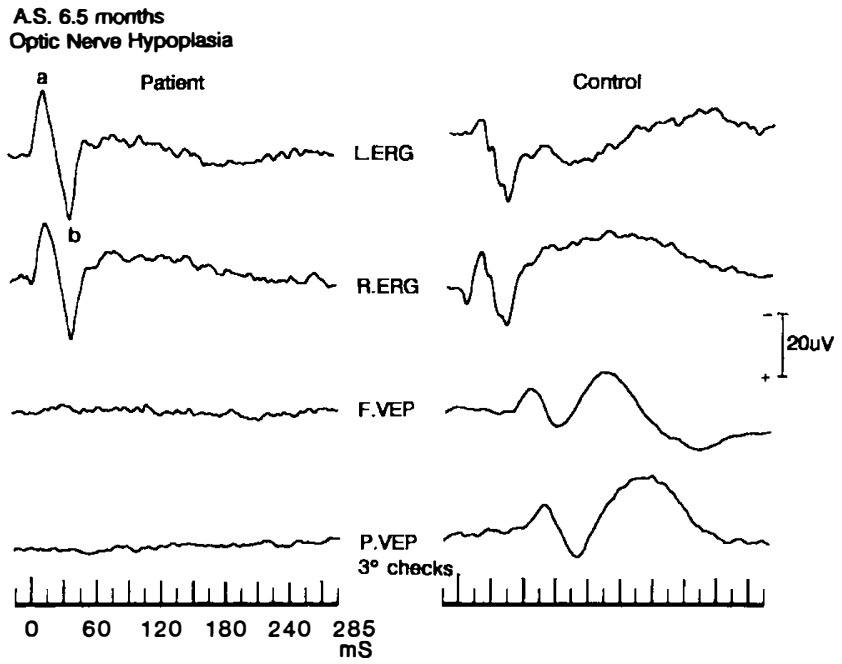

Fig. 6. Flash ERGs and VEPs to binocular flash and pattern reversal stimulation with 3 degree checks in a 6.5 month old infant with bilateral marked optic nerve hypoplasia (on left) and those from a matched control infant (on right). Note no VEP activity is detectable from the optic nerve hypoplasia patient and flash ERGs are very large.

with optic nerve hypoplasia are likely to have a normal fundus apart from the double ring sign of the optic disc which can most effectively be observed on direct ophthalmoscopy. This sign, if present, is not always easily detected, especially if the child is active and only a brief glimpse of the fundus can be obtained with an indirect ophthalmoscope. In many centres fundal examination is carried out whilst the child is anaesthetised in order to get a good stable view of the disc. Combined ERG/VEP testing will show strongly that the problem is post-retinal but it will not, of course, indicate what the pathology might be. CT scanning and regular paediatric assessments need to be carried out on these patients as optic nerve hypoplasia is often associated with anomalies of midline brain structures such as the septum pellucidum, hypothalamus and corpus callosum.

Figure 6 shows the left and right eye skin ERGs, and binocular VEPs to flash and pattern reversal stimulation from a six-month-old baby girl with marked optic nerve hypoplasia. The ERGs are large and no VEP activity is detectable either to flash or pattern stimulation with large three degree checks. $\dagger$ At the time of the recording she appeared to be behaviourally blind with roving eye movements. Both optic discs were very hypoplastic. All the other developmental milestones were normal, but her head circumference was noted to be at the 10th percentile. CT scanning confirmed small optic nerves, and showed the pituitary gland to be small also.

In segmental or less severe optic nerve hypoplasia, the VEP is attenuated and usually, though not always, of normal latency. If the hypoplasia is mild, then pattern and flash VEPs are likely to be within normal limits, although they may be mildly reduced visual acuity levels or subtle visual field defects.

$\dagger$ When nystagmus is present, it is best to use coarse checksizes for pattern reversal stimulation. ${ }^{39}$

\section{vi. Hemisphere dysfunction}

It is known from studies of half stimulation in healthy adults using pattern reversal stimulation, that the main positivity of the pattern reversal VEP (P100) is predominantly distributed over the side of scalp ipsilateral to the stimulated half-field. ${ }^{38}$ Thus for left half stimulation P100 is predominant over the left side of scalp, and vice versa when the right half field is stimulated. Over the contralateral scalp there is often a negativity of similar latency. It has been postulated that this apparently paradoxical distribution for P100 occurs because electrodes over the ipsilateral scalp are well placed to pick up activity of generators situated in the medial surface of the activated occipital cortex, contralateral to the stimulated field. ${ }^{38}$

In patients with unilateral occipital lesions associated with homonymous hemianopic defects, the pattern reversal P100 is predominantly distributed over the side of scalp ipsilateral to the preserved half-field. The distribution is the same when comparing responses from each eye, i.e. the VEP asymmetry is uncrossed..$^{32}$

We find that an uncrossed asymmetry can be observed
L.Eye

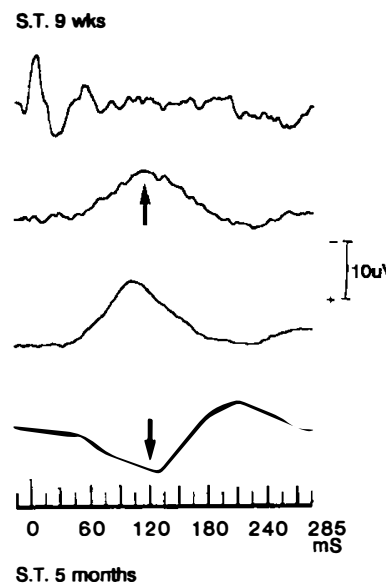

S.T. 5 months
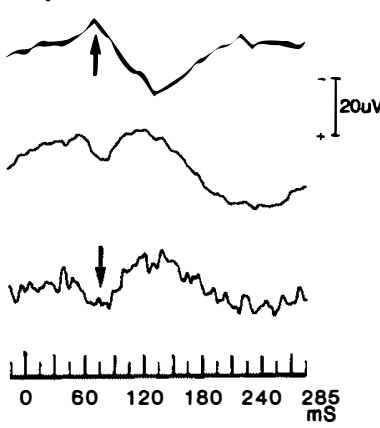

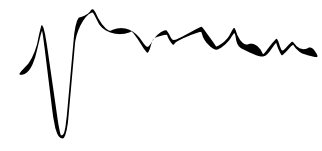

R.Eye
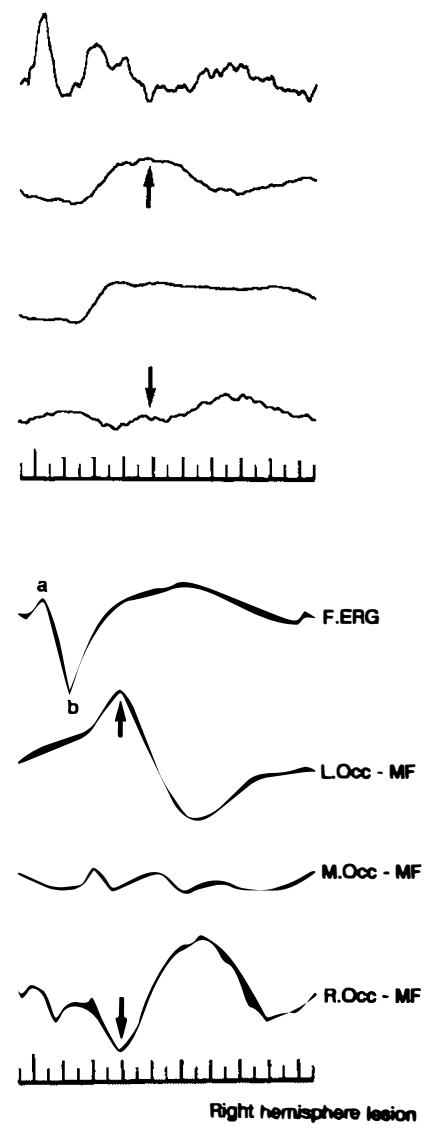

Fig. 7. ERGs and VEPs to flash stimulation in infant with right hemisphere atrophy. Infant recorded at 9 weeks and 5 months of age. ERGs recorded from skin electrodes (Sk.ERG), and VEPs recorded from electrodes at left occipital (L.Occ), mid-occipital (M.Occ) and right occipital (R.Occ) locations. For stimulation of each eye, the positivity (arrowed downwards) is recorded on the right side of the scalp suggesting a preserved right half-field response (i.e. a functional left hemisphere). 
in infants and is found for flash stimulation also, though less reliably than for pattern reversal stimulation. Figure 7 shows the asymmetrical flash VEPs in a young infant with severe hemiatrophy thought to be due to damage of one hemisphere during amniocentesis when the fetus was estimated to have a gestational age of 17 weeks. Note the marked asymmetry in the VEP when comparing the lateral electrodes. A positivity is recorded over the right scalp suggesting well preserved function of the left hemisphere. However, it appears that there is poor activity over the left side of the scalp (i.e. associated with right hemisphere dysfunction). Atrophy of the right hemisphere was clearly revealed by MRI and CT scanning. In other studies, we have described the utility of VEP recording in demonstrating dysfunction of one hemisphere in young patients with only 'soft' signs. ${ }^{40}$

\section{DISCUSSION}

Concurrent ERG/VEP recording can give very useful information and visual pathway function. Such information is especially valuable when dealing with young pre-verbal children. By helping to establish a diagnosis at an early age, it can facilitate genetic counselling and preparation for educational arrangements.

However, like most other diagnostic techniques, ERG and VEP testing have weaknesses and pitfalls. It is important to be cognisant of physiologic, stimulating and recording factors which could lead to false positive or false negative results. Stimulus and recording parameters have to be judiciously selected and carefully controlled to ensure recording conditions are optimal and reliable (see references 41, 42 for details). Excepting perhaps the electrophysiological changes associated with albinism, ERG and VEP changes associated with clinically significant visual pathway dysfunction are generally not uniquely characteristic of a particular clinical condition. The results of electrodiagnostic testing need to be considered with other clinical signs and test results, and best interpreted in the context of the suspected clinical problem.

Below is a description of some ambiguities associated with ERG/VEP testing confined to the clinical conditions mentioned above. Flash ERGs are commonly not detectable in a variety of childhood conditions associated with rod dysfunction, e.g. Refsum's syndrome, Senior's Syndrome, Alstrom's early retinitis pigmentosa and Laurence-Moon-Biedel Syndrome. VEP recording will help identify those conditions where macular function is relatively well preserved (if nystagmus is present, this may be clinically difficult to judge in a young child). We have come across the situation where, on the basis of a nonrecordable ERG, a young child was diagnosed as having Leber's Amaurosis. ERG/VEP testing in our laboratory showed no ERG activity, however, the VEPs to flash and pattern stimulation were well preserved indicating good macular function (not found in Leber's Amaurosis patients). The VEP findings, and the detection of saccade palsy, led to MRI testing which revealed hypoplasia of the cerebellar vermis and revision of the diagnosis to Joubert's syndrome.
If nystagmus is not present or conspicuous and ERG and VEP testing is not carried out, it is not uncommon for Delayed Visual Maturation to be considered as a possible diagnosis in infants of less than four months of age who appear to have poor vision. We find that the electrophysiological distinction between Leber's Amaurosis and Delayed Visual Maturation is clear-cut. In patients with uncomplicated Delayed Visual Maturation the ERG and VEP findings are normal ${ }^{27}$ whereas, as described above, in Leber's Amaurosis they are markedly abnormal. ${ }^{33}$ The presence of normal ERGs and VEPs indicates well preserved function of the primary retino-cortical pathway; however, it does not exclude the possibility of dysfunction in other areas concerned with visual processing, attention and eye movements.

Congenital cone dysfunction patients must be tested under fully darkened (scotopic) conditions. It is the presence of rod mediated activity and the absence of cone mediated activity that characterises this condition electrodiagnostically. If testing is carried out under photopic conditions (i.e. leaving room lights on and curtains undrawn), as occurs in many clinical neurophysiology departments, then patients with cone dysfunction are likely to be misdiagnosed as they will produce no ERG activity and little VEP activity, giving an electrodiagnostic picture similar to that found in patients with Leber's Amaurosis (see reference 33 for more details).

Infants with congenital cone dysfunction usually present with nystagmus and most, though not all, have some degree of photophobia. Fundal examination is usually unremarkable, however, the foveal area may not be well defined, particularly in patients with a blonde complexion. Thus the clinical picture may be similar to albinism, in which foveal hypoplasia and photophobia are usually conspicuous. ${ }^{35}$

Detection of the electrophysiological features associated with albinism relies on good technique. In infants, monocular flash stimulation gives the most reliable results. However, it is essential to ensure no light enters the covered eye as this will partially, or completely mask any albino crossed asymmetry. Similarly, if testing is only carried out binocularly, then the electrodiagnostic picture will not be specific. It will merely suggest post-retinal dysfunction, as poor VEP activity is recorded in the occipital mid-line, and lateralised occipital activity will be much reduced due to the fact that each eye is producing activity of opposite polarity. In our experience, well lateralised occipital electrodes (about $4-5 \mathrm{~cm}$ from the midline) and a monopolar montage with the reference electrode well away from occipital, temporal and parietal areas give the most reliable findings in childhood albinism. ${ }^{36}$

A multi-channel occipital recording is essential not only to identify chiasmal anomalies (e.g. associated with compression or albinism) but also to detect dysfunction of one occipital hemisphere. A monocular montage with a common reference placed away from the occipital/parietal area is also more advantageous in this context (a midfrontal location is probably the best location for the refer- 
ence electrode) ${ }^{40}$ In the adult, pattern reversal stimulation and half-field testing can be easily accomplished. Where there is a complete lateral hemianopic field defect, little or no VEP activity is recordable when stimulating the blind half-field, and a markedly asymmetrical VEP is recordable for stimulation of the preserved half-field. As explained above, the P100 of the pattern reversal VEP is apparently paradoxically distributed; it is predominantly seen over the side of scalp ipsilateral to the preserved half field. We reliably find that a young child with a unilateral hemisphere lesion involving the occipital area will produce asymmetrical pattern and flash VEPs on full-field stimulation. A VEP asymmetry is of greatest significance when there is a positivity around $100 \mathrm{~ms}$ over one side of the scalp, and a negativity of similar latency over the other side. With a monopolar montage as described above, the main positivity is 'paradoxically lateralised'. If a bipolar montage is used, with a reference electrode placed in the vicinity of the active electrodes and over the same hemisphere, then the lateralisation emphasis of the occipital asymmetry is likely to be reversed (see reference 41 for details).

We are most grateful to the Iris Fund and The Wooden Spoon Society for financial support.

Key words: Infant ERGs, Infant VEPs.

\section{REFERENCES}

1. Mann I: The Development of the Human Eye. 3rd ed. British Medical Association, 1964. London.

2. Horsten GPM and Winkelman JE: Electrical activity of the retina in relation to histological differentiation in infants born prematurely and at full-term. Vision Res 1962, 2: 269-76.

3. Abramov I, Gordon J, Hendrikson A, Hainline L, Dobson V, La Bossiere E: The retina of the newborn human infant. Science 1982, 217: 265-7.

4. Youdelis $\mathrm{C}$ and Hendrickson A: A qualitative and quantitative analysis of the human fovea during development. Vision Res 1986, 26: 847-55.

5. Nakayama K: Studies of the mylelinization of the human optic nerve. Jap J Ophthalmol 1967, 11: 18-26.

6. Magoon EH and Robb RM: Development of myelin in optic nerve and tract, a light and electron microscopic study. Arch Ophthalmol 1981, 99: 655-9.

7. Garey LJ and de Courten C: Structural development of the lateral geniculate nucleus and visual cortex in monkey and man. Behav Brain Res 1983, 10: 3-13.

8. de Courten C and Garey LJ: Morphology of the neurons in the human lateral geniculate nucleus and their normal development. Exp Brain Res 1982, 47: 159-71.

9. Purpura DP: Morphogenesis of visual cortex in the preterm infant. In: Brazier MA (ed): Growth and Development of the Brain. Raven Press, New York, 1975, pp 33-49.

10. Sauer B, Kammarat G, Kauthausen I, Kretschmann HJ, Lange HW, Wigert F: Qualitative and quantitative development of the visual cortex in man. J Comp Neuro 1983, 214: 441-50.

11. Zetterstrom B: The clinical electroretinogram IV. The electroretinogram in children during the first year of life. Acta Ophthalmol 1951, 29: 295-304.

12. Zetterstrom B: The electroretinogram in premature children. Acta Ophthalmol 1952, 30: 405-8.

13. Shipley $T$ and Anton MT: The human electroretinogram in the first day of life. J Pediatrics 1964, 65: 733-9.
14. Mactier H, Dexter JD, Hewett JE, Latham CB, Woodruff $\mathrm{CW}$ : The electroretinogram in preterm infants. $J$ Pediatrics 1988, 113: 607-12.

15. Fulton $A B$ and Hansen RM: Electroretinography: Application to clinical studies of infants. $J$ Pediat Ophthalmol Strab 1985, 22: 251-5.

16. Grose J, Harding GFA, Wilton AY, Bissenden JG: The maturation of the pattern reversal VEP and flash ERG in pre-term infants. Clin Vis Sci 1989, 4: 239-46.

17. Marmor MF, Arden GB, Nilsson S-E, Zrenner E: Standard for clinical electroretinography. Doc Ophthalmol 1989, 73: 303-11.

18. Fiorentini A, Pirchio M, Spinelli D: Development of retinal and cortical responses to pattern reversal in infants. Behav Brain Res 1983, 10: 99-106.

19. Kriss A, Hammerton M, Taylor D: Effect of defocussing on retinal and occipital responses. Electroencephal Clin Neurophysiol 1985, 61: S1-S274.

20. Ciganek ML: The EEG response (evoked potential) to light stimulation in man. Electroenceph Clin Neurophysiol 1961, 13: $165-72$.

21. Harding GFA: Origins of visual cortical potentials components. In: Heckenlively JR and Arden GB: Principles and Practice of Clinical Electrophysiology of Vision. Mosby, St. Louis, 1991, pp 132-44.

22. Barnet AB, Friedman SL, Weiss IP, Ohlrich ES, Shanks B, Lodge A: VEP development in infancy and early childhood. A longitudinal study. Electroencephal Clin Neurophysiol 1980, 49: 476-89.

23. Kurtzberg D and Vaughan HG: Electrophysiologic assessment of auditory and visual function in the newborn. Clin Perinat 1985, 12: 277-99.

24. Mushin J, Hogg CR, Dubowitz LMS, Skouteli H, Arden GB: Visual evoked responses to light emitting diode (LED) photostimulation in newborn infants. Electroencephal Clin Neurophysiol 1984, 58: 317-20.

25. Taylor MJ, Menzies R, MacMillan LJ, Whyte HE: VEPs in normal full-term and premature neonates: longitudinal versus cross-sectional data. Electroencephal Clin Neurophysiol 1987, 68: 20-7.

26. Harding GFA, Grose J, Wilton A, Bissenden JG: The pattern reversal VEP in short gestation infants. Electroencephal Clin Neurophysiol 1989, 74: 76-80.

27. Moskowitz A and Sokol S: Developmental changes in the visual system as reflected by the latency of the pattern reversal VEP. Electroencephal Clin Neurophysiol 1983, 56: $1-15$.

28. Lambert SR, Kriss A, Taylor D: Delayed Visual Maturation-A longitudinal clinical and electrophysiological assessment. Ophthalmol 1989, 96: 524-8.

29. Spekreijse H: Comparison of acuity tests and pattern evoked potential criteria: Two mechanisms underly acuity maturation in man. Behav Brain Res 1983, 10: 107-17.

30. Halliday AM, Barrett GB, Blumhardt LD, Kriss A: The macular and paramacular subcomponents of the pattern response. In: Lehmann D and Callaway E (eds): Human Evoked Potentials: Applications and Problems. Plenum Press, New York, 1979, pp 135-51.

31. Halliday AM: Evoked Potentials in Clinical Testing. Churchill Livingstone, Edinburgh, 1982.

32. Blumhardt LD, Barrett G, Halliday AM: The asymmetrical visual evoked potential to pattern reversal in one half-field and its significance for the analysis of visual field defects. Br J Ophthalmol 1917, 61: 456-61.

33. Lambert SR, Kriss A, Taylor D, Coffey R, Pembury M: Follow-up and diagnostic reappraisal of 75 patients with Leber's Congenital Amaurosis. Am J Ophthalmol 1989, 107: 624-31.

34. Guillery RW, Okoro AN, Witkop CJ: Abnormal visual pathways in the brain of a human albino. Brain Res 1975, 96: 373-7. 
35. Russell-Eggitt I, Kriss A, Taylor D: Albinism in childhood: A flash VEP and ERG study. Br J Ophthalmol 1990, 74: $136-40$.

36. Kriss A, Russell-Eggitt I, Taylor D: Childhood albinism: Visual electrophysiological features. Ophthalm Paed Genetics 1990, 11: 185-92.

37. Apakarian $P$ and Spekreijse $H$ : The VEP and misrouted pathways in albinism. In: Cracco RA and Bodis-Wollner I (eds): Evoked Potentials. Liss, New York, 1986, pp 211-22.

38. Barrett G, Blumhardt L, Halliday AM, Halliday E, Kriss A:
A paradox in the lateralisation of the visual evoked response. Nature (Lond) 1976, 261: 253-5.

39. Kriss A, Gresty M, Shawkat F, Taylor D: Effects of induced nystagmus on pattern and flash VEPs. Ophthalm Physiol Opt 1989, 9: 108.

40. Lambert SR, Kriss A, Taylor D: Detection of isolated occipital lobe anomalies during early childhood. Dev Med Child Neurol 1990, 32: 451-5.

41. Kriss A: Stimulation and recording problems. In: Halliday AM (ed): Evoked Potentials in Clinical Testing. Churchill Livingstone, Edinburgh, 1982. 